\title{
Evolving communication in embodied agents: Assessment and Open Challenges
}

\author{
Stefano Nolfi and Marco Mirolli \\ Institute of Cognitive Sciences and Technologies, CNR, Roma, Italy \\ \{stefano.nolfi,marco.mirolli\}@istc.cnr.it
}

\begin{abstract}
In this Chapter we summarize the progresses that have recently been made in the study of the emergence of communication in artificial embodied agents along different dimensions, including the understanding of the adaptive roles of communication, the expressive power and organization complexity of signalling systems, the stability, robustness, and evolvability of communication, and the knowledge gain obtained with such models. Finally, we briefly discuss which we think are the most important open challenges for future research in this area.
\end{abstract}

\section{Introduction}

In this Chapter, which concludes the Part on the evolution of communication, we briefly summarize the progresses made in the experimental research described in the previous Chapters and in other recent related researches, and we briefly discuss the issues that still represent open challanges for future research in this area. In evaluating the progresses that have been made we will proceed along the dimensions introduced in Chapter ??.

\section{Adaptive role}

The theoretical and experimental work carried out in recent research allow us to identify the characteristics of the task/environment and of the agents that might lead to the evolution of communication skills in population of initially non-communicating agents. In particular, these researches have demonstrated experimentally how communication skills tend to emerge in the following cases:

- When agents have to solve problems which require the exhibition of coordinated behaviors: e.g. coordinated and/or synchronized collective behaviors, as in Chapter ?? (see also Sperati et al., 2008; Trianni et al., 2007; Tuci et al., 2008), and collective decision behaviors, as in Chapter ??, see also Marocco and Nolfi, 2007; Uno et al., 2007).

- When agents have to solve problems which require the exhibition of cooperative behaviors: i.e. problems which can be solved individually but in which the possibility to have access to the information available to other agents or to manipulate other agents' behaviors can improve individual's and/or group's performance, as in Chapter ?? (see also Ampatzis et al., 2008; Trianni et al., 2007). 
- When communication can facilitate the learning process of individuals (Acerbi and Nolfi, 2007).

Moreover, the studies referred above demonstrate how the possibility to develop communicative skills allow agents to solve a variety of problems that could not be solved (or that could not be solved with the same level of performance) without communication.

As we mentioned above, the possibility to evolve communication skills in embodied agents which are selected for the ability to display coordinated behaviors has already been demonstrated in simulation, for example, in the pioneering works of Werner and Dyer (1992) and Di Paolo (2000). The results obtained in these studies have however been extended with respect to the complexity of the chosen task, to the ability to display multiple skills, and to the possibility to demonstrate the results on physical (or realistically simulated) robots.

\section{Expressive power and organization complexity}

Recent research has also produced significant progresses with respect to the identifications of the conditions that can lead to the evolution of communication systems with a rich expressive power and with a complex organization (to the extent that these properties are required).

With respect to the number of signals, for example, recent research works have demonstrated that evolved embodied and communicating agents can use up to 6 different functional signals (Chapter ??; see also Marocco and Nolfi, 2007).

With respect to types of signal, in the vast majority of the experiments, the signals that have emerged are: emotional/motivational or deictic (i.e. they encode information about the current state of the communicating agent or information about the current perceived environmental state of the communicating agent), non-abstract (i.e. which encode regularities which are directly available in the current sensory state), and symmetrical (i.e. in which agents do not assume different communicative roles and in which the communicating agent that sends signals to the other agents is at the same time affected by the signals that it detects from the others). This is perhaps not surprising since these are the simplest communication forms. Notably, sometimes these simple forms of communication are exploited to convey rather complex information, as is the case for the experiments reported in Marocco and Nolfi (2007), in which evolved agents exploit their signal capabilities to generate and express relational information that cannot be extracted by any individual robot alone.

In some cases, however, evolved embodied agents also display the ability to use forms of communication in which different individuals assume functionally different roles (see, for example, Chapters ??, ??, ??, and Marocco and Nolfi, 2007; Uno et al., 2007), with one agent acting as the speaker (i.e. producing a signal that affects other agents behaviour without modifying its own behaviour on the basis of the signals produced by others) and a second agent acting as the 
hearer (i.e. regulating its own behaviour on the basis of detected signals without producing signals that alter other agents behaviour).

Still other experiments have shown the emergence of abstract signals that encode either categorical information relative to the current sensory state (Uno et al., 2007) or regularities obtained by integrating sensory-motor information through time (Chapters ??, ??), see also Tuci and Ampatzis, 2007. Finally, the emergence of displaced signals (i.e. signals providing information that is independent from the current state of the signaling agent) has been observed in the experiments reported in Williams et al. (2008).

With respect to the emergence of communication protocols, some experiments showed that the evolution of effective communication can also imply the development of an ability to regulate when signals should be produced and whether detected signals should have an impact on agents behaviour or not. In particular, the experiments reported in (Trianni and Dorigo, 2006) demonstrated how the possibility to regulate when the signal are produced significantly impacts the performance of the agents even when the characteristics of the signal are predetermined by the experimenter and kept fixed during the adaptive process. Moreover, the analysis of the experiments reported in Chapter ?? indicates that the ability to react selectively to detected signals, that is on the basis of the current context, might play a crucial role for the development of an effective communication system. Finally, the experiments reported in Marocco and Nolfi, 2007) demonstrated how evolving agents can develop an ability to use different kinds of signals in functionally different contexts by selecting the appropriate communication mode on the fly. More specifically, in those experiments evolved agents relied on symmetrical communication (in which both agents concurrently act as speaker and hearer) in situations in which they need to interact bi-directionally to infer their relative status, while they used asymmetrical communication (in which one agent acts as speaker and the other acts as hearer) in situations in which one agent has access to information that is relevant for the other but not vice versa.

\section{Stability, robustness, and Evolvability}

The analysis of embodied and communicating agents also provided important evidences that can help us to understand the conditions that might favour the emergence of stable and robust communication skills, and that might lead to a progressive complexification of the communication system.

More specifically, the experiments presented in Chapter ??, in which the authors have compared the results obtained by varying the level of genetic relatedness of the colony and the level at which selection operates, have demonstrated how a robust and stable communication systems evolves when colonies are composed of genetically similar individuals and/or when selection acts at the colony level (see also Chapter ??. Moreover, the experiment reported in Mirolli and Parisi (2005) have demonstrated how the possibility for agents to access and exploit their own produced signals (in other words, the possibility to use 
communicative signals for "talking to oneself") can enhance the stability of the evolved communication system.

Regarding the robustness, the work describer in Chapter ?? showed that the ability to communicate and share information among agents might lead to agents displaying behavioural skills that are robust with respect to inter-individual differences, sensor/actuator noise, and varying initial conditions.

Finally, with respect to the evolvability of communication, the work reported in Chapter ?? (see also Marocco and Nolfi, 2006) demonstrated how the coadaptation of agents individual and social/communicative skills can lead to an incremental process in which newly developed skills create the adaptive conditions for the emergence of further skills that are based on previously developed ones. This can lead to the creation of a cascade of dependencies that force the preservation of previously developed skills. Furthermore, the combination of these factors can result in the progressive complexification of agents behavioural and communicative skills (within the limits imposed by the complexity of the experimental setup).

\section{Knowledge gain (modeling)}

In some cases the researches conducted through the synthetic method can also lead to the formulation of new theories on the evolution of communication in natural organisms or even to the generation of evidences that can be used to confirm or disconfirm existing theories.

In particular, the experiments described in Chapter ?? and ?? allowed the authors not only to provide guidelines for designing artificial communicating agents, but also to formulate predictions on the evolutionary conditions that may lead to the emergence of communication forms, including cooperative communication and deceptive signaling in biological organisms. Indeed, the synthetic approach followed in these works allows to circumvent the problem constituted by the fact that performing similar experiments on biological organisms (e.g. on insects whith elaborate social organization) is not feasible due to the long generation times of these organisms and to the difficulty of breeding them in the laboratory.

Furthermore, the experiments described in Chapter ?? (see also Mirolli and Parisi, 2008) led to the identification of a new factor that might explain the evolutionary emergence of communication. This factor, which has been named 'producer bias', consists in the tendency to spontaneously produce different signals in adaptively different contexts. The hypothesis that natural communication systems might evolve thanks to producer biases is complementary to the most commonly accepted explanations of the evolution of communication, that is those based on receiver (or sensory) biases, i.e. the exploitation, by the signalers, of behavioral biases in the receivers (Guilford and Dawkins, 1991; Ryan, 1990).

Finally, the analysis of the experiments reported in ??, provides new insights on the question of how signals are grounded on agents sensory-motor states. 
More precisely, the obtained results indicate that signals might depend not only on agents sensory states but also on agents own behaviours.

\section{Open questions for future research}

The recent work included or referred to in this book demonstrate how the synthetic methodology illustrated in Chapter ?? has allowed significant progresses in the understanding of the evolution of communication and in the development of effective methodologies for building embodied agents that are able to cooperate and communicate on the basis of a self-organized communication system. However, much more can and should be done in future research. In particular, we see two main challenges for future research.

The first challenge consists in developing new effective methods through which embodied agents can adapt both ontogenetically and phylogenetically. Such methods might in fact allow to address another important issue, that is the role of social learning and cultural evolution in the emergence of communication. For preliminary studies addressing these issues see Cangelosi (2007); Mirolli and Parisi (2005); Vogt (2006).

The second and probably most difficult challenge for future research in this are consists in developing these methods so to observe the emergencec of complex forms of communication that are structured and compositional. In this respect, a fundamental issue that we think should be systematically addressed is trying to understand which are the necessary prerequisites for the emergence of such complex forms of communication. For preliminary studies addressing these issues see Cangelosi (2007); Cangelosi and Parisi (2004). 


\section{Bibliography}

Acerbi, A. and Nolfi, S. (2007). Social learning and cultural evolution in embodied and situated agents. In Proceedings of the First IEEE Symposium on Artificial Life, pages 333-340, Piscataway, NJ. IEEE Press.

Ampatzis, C., Tuci, E., Trianni, V., and Dorigo, M. (2008). Evolution of signaling in a multi-robot system: Categorization and communication. Adaptive Behaviour, 16(1):5-26.

Cangelosi, A. (2007). Adaptive agent modeling of distributed language: Investigations on the effects of cultural variation and internal action representations. Language Sciences, 29:633-649.

Cangelosi, A. and Parisi, D. (2004). The processing of verbs and nouns in neural networks: Insights from synthetic brain imaging. Brain and Language, 89(2):401-408.

Di Paolo, E. A. (2000). Behavioral coordination, structural congruence and entrainment in a simulation of acoustically coupled agents. Adaptive Behavior, $8(1): 25-46$.

Guilford, T. and Dawkins, M. S. (1991). Receiver psychology and the evolution of animal signals. Animal Behaviour, 42:1-14.

Marocco, D. and Nolfi, S. (2006). Origins of communication in evolving robots. In Nolfi, S., Baldassarre, G., Calabretta, R., Hallam, J., Marocco, D., Miglino, O., Meyer, J.-A., and Parisi, D., editors, From animals to animats 9: Proceedings of the Ninth International Conference on Simulation of Adaptive Behaviour, volume 4095 of $L N A I$, pages 789-803, Berlin. Springer Verlag.

Marocco, D. and Nolfi, S. (2007). Emergence of communication in embodied agents evolved for the ability to solve a collective navigation problem. Connection Science, 19(1):53-74.

Mirolli, M. and Parisi, D. (2005). How can we explain the emergence of a language which benefits the hearer but not the speaker? Connection Science, 17(3-4):325-341.

Mirolli, M. and Parisi, D. (2008). How producer biases can favour the evolution of communication: An analysis of evolutionary dynamics. Adaptive Behavior.

Ryan, M. J. (1990). Sexual selection, sensory systems and sensory exploitation. Oxford Surveys in Evolutionary Biology, 7:157-195.

Sperati, V., Trianni, V., and Nolfi, S. (2008). Evolving coordinated group behaviours through maximisation of mean mutual information. Swarm Intelligence, 2-4:73-95. 
Trianni, V., Ampatzis, C., Christensen, A., Tuci, E.and Dorigo, M., and Nolfi, S. (2007). From solitary to collective behaviours: Decision making and cooperation. In et al., F. A., editor, Advances in Artificial Life. Proceedings of the 9th European Conference on Artificial Life (ECAL 2007), volume 4648 of Lecture Notes in Artificial Intelligence, pages 575-584. Springer Verlag, Berlin, Germany.

Trianni, V. and Dorigo, M. (2006). Self-organisation and communication in groups of simulated and physical robots. Biological Cybernetics, 95:213-231.

Tuci, E. and Ampatzis, C. (2007). The evolution of acoustic communication between two cooperating robots. In Almeida y Costa, F., Rocha, L., Costa, E., Harvey, I., and Coutinho, A., editors, Proceedings of the 9th European Conference on Artificial Life (ECAL 2007), volume 4648 of LNAI, pages 395404, Berlin. Springer Verlag.

Tuci, E., Ampatzis, C., Vicentini, F., and Dorigo, M. (2008). Evolving homogeneous neuro-controllers for a group of heterogeneous robots: coordinated motion, cooperation, and acoustic communication. Artificial Life, 14(2):157178.

Uno, R., Marocco, D., Nolfi, S., and Ikegami, T. (2007). Transition from imperatives to declara-tives in artificial communicating systems. In Acerbi, A., Marocco, D., and Vogt, P., editors, Proceeding of the workshop on Social Learning in Embodied Agents.

Vogt, P. (2006). Cumulative cultural evolution: can we ever learn more? In Nolfi, S., Baldassarre, G., Calabretta, R., Hallam, J., Marocco, D., Miglino, O., Meyer, J.-A., and Parisi, D., editors, From Animals to Animats 9: Proceedings of the Ninth International Conference on Simulation of Adaptive Behaviour, pages 738-749, Berlin. Springer Verlag.

Werner, G. M. and Dyer, M. G. (1992). Evolution of communication in artificial organisms. In Langton, C., Taylor, C., Farmer, D., and Rasmussen, S., editors, Artificial Life II, pages 659-687. Addison-Wesley, Redwood City, CA.

Williams, P., Beer, R., and Gasser, M. (2008). Evolving referential communication in embodied dynamical agents. In Bullock, S., Noble, J., Watson, R., and Bedau, M., editors, Artificial Life XI: Proceedings of the Eleventh International Conference on the Simulation and Synthesis of Living Systems, pages 707-709, Cambridge, MA. MIT Press. 